Pacific Journal of Mathematics

ON THE CONVERGENCE OF QUASI-HERMITE-FEJÉR

K. K. MATHUR AND R. B. SATE 


\title{
ON THE CONVERGENCE OF QUASI-HERMITE-FEJÉR INTERPOLATION
}

\author{
K. K. Mathur and R. B. Saxena
}

The present paper deals with the convergence of quasiHermite-Fejér interpolation series $\left\{S_{n}(x, f)\right\}$ satisfying the conditions

$S_{n}(1, f)=f(1), S_{n}\left(x_{n \nu}, f\right)=f\left(x_{n \nu}\right) 1 \leqq \nu \leqq n, S_{n}(-1, f)=f(-1)$

and

$$
S_{n}^{\prime}\left(x_{n \nu}, f\right)=\beta_{n \nu} \quad 1 \leqq \nu \leqq n,
$$

where $\beta_{n \nu}$ 's are arbitrary numbers; $x_{n 0}=1, x_{n, n+1}=-1$ and $\left\{x_{n \nu}\right\}$ are the zeros of orthogonal polynomial system $\left\{p_{n}(x)\right\}$ belonging to the weight function $\left(1-x^{2}\right)^{p}|x|^{q}, 0<p \leqq \frac{1}{2}$, $0<q<1$ (which actually vanishes at a point in the interval $[-1,+1])$. Further it has been proved that quasi-conjugate pointsystem $\left\{X_{n \nu}\right\}$ (similar to Fejér conjugate pointsystem) belonging to the fundamental pointsystem $\left\{x_{n_{\nu}}\right\}$ lie everywhere thickly in the interval $[-1,+1]$.

Let there be given a point system

$$
\begin{aligned}
1=x_{n 0}>x_{n 1}>x_{n 2}>\cdots>x_{n n}>x_{n, n+1}=-1 \\
\quad(n=1,2, \cdots)
\end{aligned}
$$

on the real axis and arbitrary real numbers

$$
\begin{gathered}
y_{n 0}, y_{n 1}, y_{n 2}, \cdots, y_{n n}, y_{n, n+1}, \\
y_{n 1}^{*}, y_{n 2}^{*}, \cdots, y_{n n}^{*} .
\end{gathered}
$$

Then setting

$$
\omega_{n}(x)=c_{n}\left(x-x_{n 1}\right)\left(x-x_{n 2}\right) \cdots\left(x-x_{n n}\right) \quad\left(c_{n} \neq 0\right)
$$

and

$$
l_{n \nu}(x)=\frac{\omega_{n}(x)}{\omega_{n}^{\prime}\left(x_{n \nu}\right)\left(x-x_{n \nu}\right)}(\nu=1,2, \cdots, n),
$$

the quasi-Hermite-Fejér interpolation polynomial $S_{n}(x)$ [6] is given by

$$
S_{n}(x)=\sum_{\nu=0}^{n+1} y_{n \nu} r_{n \nu}(x)+\sum_{\nu=1}^{n} y_{n \nu}^{*} \rho_{n \nu}(x)
$$

where $r_{n \nu}(x)$ and $\rho_{n \nu}(x)$ are called the fundamental polynomials of the 1st and the second kind of quasi-Hermite-Fejér interpolation.

For the fundamental polynomials of the 1st kind we have 


$$
\begin{aligned}
r_{n 0}(x) & =\frac{1+x}{2} \cdot \frac{\omega_{n}(x)^{2}}{\omega_{n}(1)^{2}}, \\
r_{n, n+1}(x) & =\frac{1-x}{2} \cdot \frac{\omega_{n}(x)^{2}}{\omega_{n}(-1)^{2}}, \\
r_{n \nu}(x) & =\frac{1-x^{2}}{1-x_{n \nu}^{2}} v_{n \nu}(x) l_{n \nu}(x)^{2}, \quad(\nu=1,2, \cdots n)
\end{aligned}
$$

where

$$
\begin{aligned}
& v_{n \nu}(x)=1+c_{n \nu}\left(x-x_{n \nu}\right), \\
& c_{n \nu}=\frac{2 x_{n \nu}}{1-x_{n \nu}^{2}}-\frac{\omega_{n}^{\prime \prime}\left(x_{n \nu}\right)}{w_{n}^{\prime}\left(x_{n \nu}\right)} \quad(\nu=1,2, \cdots, n)
\end{aligned}
$$

and those of second kind

$$
\rho_{n \nu}(x)=\frac{1-x^{2}}{1-x_{n \nu}^{2}}\left(x-x_{n \nu}\right) l_{n \nu}(x)^{2} \quad(\nu=1,2, \cdots, n) .
$$

The polynomials $S_{n}(x)$ are the unique polynomials of degree $\leqq 2 n+1$ which satisfy the requirements:

$$
\begin{array}{ll}
S_{n}\left(x_{n \nu}\right)=y_{n \nu} & \nu=0,1,2, \cdots, n+1, \\
S_{n}^{\prime}\left(x_{n \nu}\right)=y_{n \nu}^{*} & \nu=1,2, \cdots, n .
\end{array}
$$

From the unicity of the polynomials $S_{n}(x)$ it follows that for each polynomial $\Pi(x)$ of degree $\leqq 2 n$

$$
\Pi(x)=\sum_{\nu=0}^{n+1} \Pi\left(x_{n \nu}\right) r_{n \nu}(x)+\sum_{\nu=1}^{n} \Pi^{\prime}\left(x_{n \nu}\right) \rho_{n \nu}(x)
$$

holds. For the special case $\Pi(x) \equiv 1$, we have

$$
\sum_{\nu=0}^{n+1} r_{n \nu}(x) \equiv 1
$$

2. Let $f(x)$ be continuous in $-1 \leqq x \leqq 1$ and $f\left(x_{n \nu}\right)$ its values at the points $x_{n \nu}(\nu=0,1,2, \cdots, n+1)$. Further let $y_{n \nu}^{*}(\nu=1,2, \cdots n)$ be arbitrary real numbers then the polynomials $S_{n}(x)$ in (1.5) written as

$$
S_{n}(x, f)=\sum_{\nu=0}^{n+1} f\left(x_{n \nu}\right) r_{n \nu}(x)+\sum_{\nu=0}^{n} y_{n \nu}^{*} \rho_{n \nu}(x)
$$

are called the generalised quasi-Hermite-Fejer interpolation polynomials. For $y_{n \nu}^{*}=0$, they are called quasi-step parabolas. In this case for $\omega_{n}(x)=P_{n}(x)$, where $P_{n}(x)$ stands for the $n$th Legendre polynomial, 
the interpolatory polynomials

$$
\begin{aligned}
R_{n}(x)= & f(1) \frac{1+x}{2} P_{n}(x)^{2}+f(-1) \frac{1-x}{2} P_{n}(x)^{2} \\
& +\sum_{\nu=1}^{n} f\left(x_{n \nu}\right) \frac{1-x^{2}}{1-x_{n \nu}^{2}}\left(\frac{P_{n}(x)}{P_{n}^{\prime}\left(x_{n \nu}\right)\left(x-x_{n \nu}\right)}\right)^{2}
\end{aligned}
$$

have been obtained by E. Egerváry and P. Turán [2]. They have shown that if $f(x)$ is a function continuous in the closed interval $[-1,1]$, then the polynomials in (2.2) converge uniformly to $f(x)$ in $[-1,1]$. The convergence of the polynomials $S_{n}(x, f)$ in $(2.1)$ constructed on the roots of $P_{n}(x)$ has been investigated by P. Szász [6]. He has shown that assuming $f(x)$ to be continuous and $\left|y_{n \nu}^{*}\right|<\Delta$, where $\Delta$ is a constant independent of $n$ and $\nu$ the series $S_{n}(x, f)$ in $(2.1)$ converges uniformly to $f(x)$ in $[-1,1]$.

In this paper we answer the question of P. Turán for the quasiHermite-Fejér interpolation polynomials $S_{n}(x, f)$ which Balázs has answered [1] in the case of Hermite-Fejer interpolation polynomials.

Does there exist in $[-1,1]$ an orthogonal polynomial system $\left\{g_{n}(x)\right\}$ whose weight function vanishes some where in this interval while the series $\left\{S_{n}(x, f)\right\}$ in (2.1) constructed on the roots of $\left\{g_{n}(x)\right\}$ converges uniformly to the continuous function $f(x)$ in the closed interval $[-1,1]$ provided $\left\{y_{n \nu}^{*}\right\}$ are bounded?

The answer to this question is explained in our Theorem 1.

3. Similar to the normal and strongly normal point system due to L. Fejér [3, 4], the notion of quasi-normal and strongly quasi-normal point systems have been defined by Szász [6]. Thus an infinite sequence of point system,

$$
x_{n 1}, x_{n 2}, \cdots, x_{n n}, \quad(n=1,2, \cdots)
$$

lying in $-1<x<1$, is called strongly quasi-normal if by the notation of (1.3) and (1.7)

$$
\begin{aligned}
1+c_{n \nu}\left(x-x_{n \nu}\right) \geqq \rho>0, \quad-1 \leqq x \leqq 1 \\
\quad(\nu=1,2, \cdots, n ; n=1,2, \cdots)
\end{aligned}
$$

where $\rho$ is a positive number independent of $x, \nu$ and $n$.

If $X_{n \nu}$ indicates a zero of $v_{n \nu}(x)$ in (1.7), then

$$
X_{n \nu}=x_{n \nu}+\frac{1}{c_{n \nu}}, \quad \nu=1,2, \cdots, n .
$$

These points will be called quasi-conjugate points similar to the conjugate points due to L. Fejér [4]. The quasi-conjugate points lie outside $[-1,1]$ when the fundamental point system is quasi-strongly 
normal. In this connection we shall answer another question of $\mathrm{P}$. Turán for the case of quasi-Hermite-Fejér interpolation polynomials which Balázs [1] has answered for the Hermite-Fejér interpolation polynomials.

Is it possible to assume in the interval $[-1,1]$ a fundamental point system whose quasi-conjugate points (3.3) lie thickly in $[-1,1]$ and the interpolation series $\left\{S_{n}(x, f)\right\}$ belonging to this fundamental point-system converges uniformly to the continuous function $f(x)$ in $[-1,1]$ provided the numbers $\left\{y_{n \nu}^{*}\right\}$ are bounded.

In Theorem II we answer this in affirmative.

4. K. V Laščenov [5] has defined orthogonal polynomials

$$
p_{n}^{(p, q)}(x)=\alpha_{n} x^{n}+\alpha_{n-2} x^{n-2}+\cdots, \quad \alpha_{n} \neq 0, p>-1, q>-1
$$

over the interval $[-1,1]$ with respect to the weight function $\left(1-x^{2}\right)^{p}|x|^{q}$ which are constant multiples of

$$
p_{n}^{(p, q)}(x)^{1}=\left\{\begin{array}{l}
P_{m}^{(p, q-1 / 2)}\left(2 x^{2}-1\right), n=2 m \\
x P_{m}^{\langle p, q+1 / 2 ;}\left(2 x^{2}-1\right), n=2 m+1
\end{array}\right.
$$

$P_{n}^{(\alpha, \beta)}(t)$ being the classical Jacobi polynomial of degree $n$ with parameters $\alpha$ and $\beta$ satisfying the differential equation

$$
\left(1-t^{2}\right) y^{\prime \prime}+[\beta-\alpha-(\alpha+\beta+2) t] y^{\prime}+n(n+\alpha+\beta+1) y=0 .
$$

The position of the roots of (4.1) is given by

$$
\begin{aligned}
-1<x_{n m+1}<x_{n m+2}<\cdots<x_{n n}<0<x_{n 1}<\cdots<x_{n m}<1 \\
\text { for } n=2 m
\end{aligned}
$$

and

$$
\begin{array}{r}
\text { (4.4) }-1<x_{n m+2}<x_{n m+3}<\cdots<x_{n n}<0=x_{n m+1}<x_{n 1}<\cdots<x_{n m}<1 \\
\text { for } n=2 m+1 .
\end{array}
$$

Since the roots are symmetrical, we have

$$
x_{n \nu}+x_{n, n+1-\nu}=0, \nu=1,2, \cdots[n / 2] .
$$

We shall prove the following:

THEOREM 1. The quasi-Hermite-Fejér interpolation series $\left\{S_{n}(x, f)\right\}$, constructed on the point system

$$
1=x_{n 0}, x_{n 1}, \cdots x_{n, n-1}, x_{n, n}, x_{n n+1}=-1 \quad n=1,2, \cdots
$$

${ }^{1}$ From now onward we shall write $p_{n}(x)$ to mean $p_{n}^{(p, q)}(x)$. 
where $x_{n \nu}(\nu=1,2, \cdots, n)$ are the zeros of the orthogonal polynomial system belonging to the weight function ${ }^{2}$

$$
\left(1-x^{2}\right)^{p}|x|^{q} \quad 0<p \leqq \frac{1}{2}, 0<q<1,
$$

converges uniformly to the continuous function $f(x)$ in $[-1,1]$ when $\left|y_{n \nu}^{*}\right| \leqq c n^{\eta}, 1>\delta / 2>\eta \geqq 0$ and $\delta=\min (2 p, q)$.

THEOREM 2. The quasi-conjugate points (3.3)

$$
x_{n \nu}=x_{n \nu}+\frac{1}{c_{n \nu}} \quad \nu=1,2, \cdots n ; n=1,2, \cdots,
$$

belonging to the fundamental point system (4.5) lie thickly in the interval $[-1,1]$.

5. Preliminaries. We shall use some well-known facts about Jacobi polynomials. We have

$$
\begin{aligned}
P_{m}^{(\alpha, \beta)}(1) & =\left(\begin{array}{c}
m+\alpha \\
m
\end{array}\right) \\
P_{m}^{(\alpha, \beta)}(-1) & =(-1)^{m} P_{m}^{(\alpha, \beta)}(1)=(-1)^{m}\left(\begin{array}{c}
m+\beta \\
m
\end{array}\right) \\
P_{m}^{(\alpha, \beta)}(t) & =(-1)^{m} P_{m}^{(\beta, \alpha)}(-t) .
\end{aligned}
$$

Further we have for $-1<x<1$

$$
P_{m}^{(\alpha, \beta)}(x)=O\left(n^{-1 / 2}\right), \alpha, \beta>-1
$$

$$
P_{m}^{(\alpha+1, \beta)}(x)=\frac{2}{(2 m+\alpha+\beta+2)} \frac{(m+\alpha+1) P_{m}^{(\alpha, \beta)}(x)-(m+1) P_{m-1}^{(\alpha, \beta)}(x)}{(1-x)}
$$

and

$$
\frac{d}{d t} P_{m}^{(\alpha, \beta)}(t)=\frac{1}{2}(m+\alpha+\beta+1) P_{m-1}^{(\alpha+1, \beta+1)}(t) .
$$

Further let $t_{\nu}=\cos \theta_{\nu}$ be the root of the polynomial

$$
P_{m}^{(\alpha, \beta)}(t)=P_{m}^{(\alpha, \beta)}(\cos \theta)
$$

then for $-1 / 2 \leqq \alpha \leqq 1 / 2,-1 / 2 \leqq \beta \leqq 1 / 2$,

$$
\frac{2 \nu-1}{2 m+1} \pi \leqq \theta_{\nu} \leqq \frac{2 \nu}{2 m+1} \pi \quad(\nu=1,2, \cdots, m) .
$$

\footnotetext{
${ }^{2}\left(1-x^{2}\right)^{p}|x|^{q}$ for $0<p \leqq \frac{1}{2}, 0<q<1$, actually vanishes at $x=0$.
} 
For $0<\theta_{2} \leqq \pi / 2$ we have

$$
P_{m}^{\prime(\alpha, \beta)}\left(\cos \theta_{\nu}\right) \geqq c_{1} \nu^{-\alpha-3 / 2} m^{\alpha+2}
$$

where $c_{1}$ is positive numerical constant.

6. In this section we shall obtain certain estimations for the polynomial $p_{n}(x)$.

We shall first prove:

Lemma 6.1. For $-1 \leqq x \leqq 1$ we have

$$
\left(1-x^{2}\right) p_{n}^{2}(x)=0\left(n^{-1}\right) \text {. }
$$

Proof of this lemma follows at once from (4.1) using (5.4).

LeMma 6.2. For the roots $x_{n \nu}\left(\nu=1,2, \cdots,\left[\frac{n}{2}\right], n=1,2, \cdots\right.$ of the polynomial $p_{n}(x)$, we have

$$
x_{n \nu}^{2}\left(1-x_{n \nu}^{2}\right) \geqq \frac{\nu^{2}}{4 n^{2}}
$$

Proof. Let $2 x_{n \nu}^{2}-1=\cos \theta_{n \nu}$, then $\left(1-x_{n \nu}^{2}\right)=\sin ^{2} \theta_{n \nu} / 2$, and $x_{n \nu}^{2}=\cos ^{2} \theta_{n \nu} / 2$. Hence

$$
x_{n \nu}^{2}\left(1-x_{n \nu}^{2}\right)=\frac{4}{4} \cos ^{2} \frac{\theta_{n \nu}}{2} \sin ^{2} \frac{\theta_{n \nu}}{2}=\frac{1}{4} \sin ^{2} \theta_{n \nu} .
$$

But from (5.7) we have

$$
\theta_{n \nu} \geqq \frac{\nu+\frac{1}{2}}{n+\frac{1}{2}} \pi>\frac{\nu \pi}{2 n}
$$

which gives

$$
\left|\sin \theta_{n \nu}\right|>\left|\sin \frac{\nu \pi}{2 n}\right|>\frac{\nu}{n}
$$

Therefore

$$
x_{n \nu}^{2}\left(1-x_{n \nu}^{2}\right)=\frac{1}{4} \sin ^{2} \theta_{n \nu}>\frac{\nu^{2}}{4 n^{2}} .
$$

7. We shall need the following lemmas for the estimation of the fundamental polynomials of the first kind. 
LEMMA 7.1. Let $x_{n \nu}$ be a root of $p_{n}(x)$, then

(i) $\frac{p_{n}^{\prime \prime}\left(x_{n \nu}\right)}{p_{n}^{\prime}\left(x_{n \nu}\right)}=\frac{2}{x_{n \nu}}\left[(p+1) \frac{x_{n \nu}^{2}}{\left(1-x_{n \nu}^{2}\right)}-\frac{q}{2}\right]$

except when $n=2 m+1$, and $\nu=m+1$. In this case we have

(ii) $\frac{p_{n}^{\prime \prime}\left(x_{n \nu}\right)}{p_{n}^{\prime}\left(x_{n \nu}\right)}=0$.

Proof. It follows from (4.1) by differentiating with respect to $x$, for $n=2 m$

$$
\frac{p_{n}^{\prime \prime}\left(x_{n \nu}\right)}{p_{n}^{\prime}\left(x_{n \nu}\right)}=4_{n \nu}\left\{\frac{\frac{d^{2}}{d t^{2}} P_{m}^{(p,(q-1 / 2))}(t)}{\frac{d}{d t} P_{m}^{(p,(q-1 / 2))}(t)}\right\}_{t=2 x_{n \nu}^{2}-1}+\frac{1}{x_{n \nu}} .
$$

By the substitution $t=2 x^{2}-1, \alpha=p, \beta=q-1 / 2$, and $n=m$, the differential equation (4.2) gives

$$
\begin{aligned}
& \left\{\frac{\frac{d^{2}}{d t^{2}} P_{m}^{(p,(q-1 / 2)}(t)}{\frac{d}{d t} P_{m}^{(p,(q-1 / 2))}(t)}\right\}_{t=2 x_{n \nu}^{2}-1} \\
& \quad=\frac{1}{4 x_{n \nu}^{2}\left(1-x_{n \nu}^{2}\right)}\left[-2(p+1)+(2 p+q+3)\left(1-x_{n \nu}^{2}\right)\right] .
\end{aligned}
$$

Substituting (7.2) in (7.1) we get

$$
\frac{p_{n}^{\prime \prime}\left(x_{n \nu}\right)}{p_{n}^{\prime}\left(x_{n \nu}\right)}=\frac{2}{x_{n \nu}}\left[(p+1) \frac{x_{n \nu}^{2}}{1-x_{n \nu}^{2}}-\frac{q}{2}\right]
$$

If however, $n=2 m+1$ and $\nu \neq m+1$, then it follows on account of (4.1) and (4.4) that

$$
\frac{p_{n}^{\prime \prime}\left(x_{n \nu}\right)}{p_{n}^{\prime}\left(x_{n \nu}\right)}=4 x_{n \nu}\left\{\frac{\frac{d^{2}}{d t^{2}} P_{m}^{(p,(q+1 / 2))}(t)}{\frac{d}{d t} P_{m}^{(p,(q+1 / 2))}(t)}\right\}_{t=2 x_{n \nu}^{2}-1}+\frac{3}{x_{n \nu}} .
$$

But from (4.2) by putting $t=2 x^{2}-1, \alpha=p, \beta=q+1 / 2$ and $n=m$ we get 


$$
\begin{aligned}
& \left\{\frac{\frac{d^{2}}{d t^{2}} P_{m}^{(p,(q+1 / 2))}(t)}{\frac{d}{d t} P_{m}^{(p,(q+1 / 2))}(t)}\right\}_{t=2 x_{n \nu}^{2},-1} \\
& \quad=-\frac{1}{4 x_{n \nu}^{2}\left(1-x_{n \nu}^{2}\right)}\left[-2(p+1)+(2 p+q+5)\left(1-x_{n \nu}^{2}\right)\right]
\end{aligned}
$$

substituting (7.5) in (7.4) we get

$$
\frac{p_{n}^{\prime \prime}\left(x_{n \nu}\right)}{p_{n}^{\prime}\left(x_{n \nu}\right)}=\frac{2}{x_{n \nu}}\left[(p+1) \frac{x_{n \nu}^{2}}{1-x_{n \nu}^{2}}-\frac{q}{2}\right] .
$$

In case $n=2 m+1$ and $\nu=m+1, x_{n \nu}=0$ on account of (4.4). But the polynomial $p_{n}(x)$ is an odd function of $x$, therefore $p_{n}^{\prime \prime}\left(x_{n \nu}\right)=0$ and in this case

$$
\frac{p_{n}^{\prime \prime}\left(x_{n \nu}\right)}{p_{n}^{\prime}\left(x_{n \nu}\right)}=0
$$

8. Estimation of the fundamental polynomials of the first kind.

Lemma 8.1. For $-1 \leqq x \leqq 1$, we have

$$
\sum_{\nu=0}^{n+1}\left|r_{n \nu}(x)\right|=O(1) \text {. }
$$

Proof. From (1.7), (1.8) and Lemma 7.1 we get for $1 \leqq \nu \leqq n$

$$
v_{n \nu}(x)=1-\frac{2}{x_{n \nu}}\left\{\frac{p x_{n \nu}^{2}}{\left(1-x_{n \nu}^{2}\right)}-\frac{q}{2}\right\}\left(x-x_{n \nu}\right) .
$$

From the representation (4.4) of $x_{n \nu}$ 's it is clear that for $n=$ $2 m+1$, and $\nu=m+1, x_{n m+1}=0$. Whence from Lemma 7.1 (ii) and (1.7) it follows that

$$
v_{n m+1}(x) \equiv 1 .
$$

For $x=0$ it follows from (8.2) on account of $0<q<1$ and $0<p \leqq \frac{1}{2}$ that

$$
v_{n \nu}(0)=1+\frac{2 p x_{n \nu}^{2}}{\left(1-x_{n \nu}^{2}\right)}-q \geqq 1-q>0 .
$$

This inequality is also applicable on account of (8.3) when $n=$ $2 m+1$, and $\nu=m+1$. For $-1<x \leqq 0$ and $x_{n \nu} \leqq 0$ we have on 
account of $v_{n \nu}\left(x_{n \nu}\right)=1$ and (8.4)

$$
v_{n \nu}(x) \geqq 1-q>0
$$$$
(0<q<1) .
$$

Since $v_{n \nu}(x)$ is a linear function in the interval $0 \leqq x<1$ it follows from $v_{n \nu}\left(x_{n \nu}\right) \equiv 1$ and $x_{n \nu} \geqq 0$ that

$$
v_{n \nu}(x) \geqq 1-q>0 \quad \text { since } 0<q<1 .
$$

We shall now prove the inequality (8.1) in the interval $-1<x \leqq 0$. In this interval $r_{n \nu}(x) \geqq 0$ for $x_{n \nu} \leqq 0$. Also $r_{n 0}(x)$ and $r_{n, n+1}(x)$ are positive. Hence from (1.12)

$$
\begin{aligned}
\sum_{\nu=0}^{n+1}\left|r_{n \nu}(x)\right| & =\sum_{x_{n \nu} \leq 0}\left|r_{n \nu}(x)\right|+\sum_{x_{n \nu}>0}\left|r_{n \nu}(x)\right| \\
& =\sum_{x_{n \nu \leqq 0}} r_{n \nu}(x)+\sum_{x_{n \nu>0}}\left|r_{n \nu}(x)\right| \\
& =1-\sum_{x_{n \nu}>0} r_{n \nu}(x)+\sum_{x_{n \nu}>0}\left|r_{n \nu}(x)\right| \\
& \leqq 1+2 \sum_{x_{n \nu}>0}\left|r_{n \nu}(x)\right| .
\end{aligned}
$$

On account of (8.2), (1.6) and (1.4) we obtain

$$
\begin{gathered}
\sum_{x_{n \nu}>0}\left|r_{n \nu}(x)\right|=\sum_{x_{n \nu}>0} \frac{1-x^{2}}{1-x_{n \nu}^{2}}\left|1-\frac{2}{x_{n \nu}}\left\{\frac{p x_{n \nu}^{2}}{1-x_{n \nu}^{2}}-\frac{q}{2}\right\}\left(x-x_{n \nu}\right)\right| \\
\times \frac{p_{n}^{2}(x)}{p_{n}^{\prime 2}\left(x_{n \nu}\right)\left(x-x_{n \nu}\right)^{2}} \\
\leqq \\
\quad \sum_{x \nu>0} \frac{1-x^{2}}{1-x_{n \nu}^{2}} \cdot \frac{p_{n}^{2}(x)}{p_{n}^{\prime 2}\left(x_{n \nu}\right)\left(x-x_{n \nu}\right)^{2}} \\
\quad+2 p \sum_{x_{n \nu}>0} \frac{\left(1-x^{2}\right) p_{n}^{2}(x)}{\left|x_{n \nu}\right|\left(1-x_{n \nu}^{2}\right)^{2} p_{n}^{\prime 2}\left(x_{n \nu}\right)\left(x-x_{n \nu}\right)} \\
\quad+(2 p+q) \sum_{x_{n \nu}>0} \frac{1-x^{2}}{\left(1-x_{n \nu}^{2}\right)} \frac{1}{\left|x_{n \nu}\right|} \cdot \frac{p_{n}^{2}(x)}{p_{n}^{\prime 2}\left(x_{n \nu}\right)\left|x-x_{n \nu}\right|} .
\end{gathered}
$$

Since $-1<x \leqq 0$ and $0<x_{n \nu}<1$, therefore $\left|x-x_{n \nu}\right|>\left|x_{n \nu}\right|$. Hence from (8.8),

$$
\begin{aligned}
\sum_{x_{n \nu}>0}\left|r_{n \nu}(x)\right| \leqq & (1+2 p+q) \sum_{x_{n \nu}>0} \frac{1-x^{2}}{1-x_{n \nu}^{2}} \cdot \frac{1}{x_{n \nu}^{2}} \cdot \frac{p_{n}^{2}(x)}{p_{n}^{\prime 2}\left(x_{n \nu}\right)} \\
& +2|p| \sum_{x n_{\nu}>0} \frac{\left(1-x^{2}\right)}{\left(1-x_{n \nu}^{2}\right)^{2} x_{n \nu}^{2}} \frac{p_{n}^{2}(x)}{p_{n}^{\prime 2}\left(x_{n \nu}\right)} .
\end{aligned}
$$

Owing to (4.1) we have

$$
p_{n}^{\prime}\left(x_{n \nu}\right)= \begin{cases}4 x_{n \nu} P_{m}^{\prime(p, 2(q-1 / 2))}\left(2 x_{n \nu}-1\right) & \text { for } n=2 m \\ 4 x_{n \nu}^{2} P_{m}^{\prime(p, 2(q+1 / 2))}\left(2 x_{n \nu}-1\right) & \text { for } n=2 m+1 .\end{cases}
$$

Thus for $n=2 m$, using (8.9) and (8.10); for $n$ odd using (8.8), (8.10) and $x^{2}<\left(x-x_{n \nu}\right)^{2}$, we have 


$$
\begin{aligned}
& \sum_{x_{n \nu}>0}\left|r_{n \nu}(x)\right| \\
& \leqq
\end{aligned}\left\{\begin{array}{r}
\frac{1}{16}(1+4 p+q) \sum_{x_{n \nu}>0} \frac{\left(1-x^{2}\right)\left[P_{m}^{(p,(q-1 / 2))}\left(2 x^{2}-1\right)\right]^{2}}{x_{n \nu}^{4}\left(1-x_{n \nu}^{2}\right)^{2}\left[\frac{d}{d t} P_{m}^{(p,(q-1 / 2))}(t)\right]_{t=2 x_{n \nu}^{2}-1}^{2}} \\
\text { for } n=2 m \\
\frac{1}{16}(1+4 p+q) \sum_{x_{n \nu}>0} \frac{\left(1-x^{2}\right)}{\left(1-x_{n \nu}^{2}\right)^{2} x_{n \nu}^{5}} \cdot \frac{\left[P_{m}^{(p,(q+1 / 2))}\left(2 x^{2}-1\right)\right]^{2}}{\left[\frac{d}{d t} P_{m}^{(p,(q+1 / 2))}(t)\right]_{t=2 x_{n \nu}^{2}-1}^{2}} \\
\text { for } n=2 m+1 .
\end{array}\right.
$$

Now Lemmas 6.1 and 6.2, with (5.8) give

$$
\sum_{x_{n \nu}>0}\left|r_{n \nu}(x)\right|=\left\{\begin{array}{r}
{\left[\sum_{\nu=1}^{m} O\left(n^{-1}\right) \frac{n^{4}}{\nu^{4}} \cdot \frac{\nu^{2 p+3}}{n^{2 p+4}}+\sum_{\nu=1}^{m} O\left(n^{-1}\right) \frac{n^{4}}{\nu^{4}} \cdot \frac{\nu^{q+2}}{n^{q+3}}\right]} \\
\text { for } n=2 m \\
{\left[\sum_{\nu=1}^{m} O\left(n^{-1}\right) \frac{n^{5}}{\nu^{5}} \cdot \frac{\nu^{2 p+3}}{n^{2 p+4}}+\sum_{\nu=1}^{m} O\left(n^{-1}\right) \frac{n^{5}}{\nu^{5}} \cdot \frac{\nu^{q+4}}{n^{q+5}}\right]} \\
\text { for } n=2 m+1
\end{array}\right.
$$

and since $0<p \leqq \frac{1}{2}, 0<q<1$, (8.12) gives

$$
\sum_{x_{n \nu}>0}\left|r_{n \nu}(x)\right|=O(1) .
$$

By a similar reasoning we can obtain for the interval $0 \leqq x<1$ and $x_{n \nu} \geqq 0$, that

$$
\sum_{x_{n \nu}<0}\left|r_{n \nu}(x)\right|=O(1) .
$$

Hence from (8.13) and (8.14) we get the lemma for $1 \leqq \nu \leqq n$, and $-1<x<1$. For $\nu=0$ and $n+1$ it is easy to see from (1.6) with $\omega_{n}(x)=p_{n}(x)$ and $(5.4)$ that

$$
r_{n 0}(x)=O(1) \quad \text { and } \quad r_{n, n+1}(x)=O(1) .
$$

At $x= \pm 1$, the lemma is trivial.

9. Estimation of the fundamental polynomials of the second kind. In this section we shall estimate the quantity

$$
\sum_{\nu=1}^{n}\left|\rho_{n \nu}(x)\right| \text {. }
$$

We shall prove the following:

Lemma 9.1. For $-1 \leqq x \leqq 1$ and $n=1,2, \cdots$ we have 


$$
\sum_{\nu=1}^{n}\left|\rho_{n \nu}(x)\right|=O\left(n^{-\delta / 2}\right), \text { where } \delta=\min (2 p, q)>0 .
$$

Proof. From (1.9) and (1.4) with $\omega_{n}(x)=p_{n}(x)$

$$
\sum_{\nu=1}^{n} \rho_{n \nu}(x)=\sum_{\nu=1}^{n}\left(x-x_{n \nu}\right) \frac{1-x^{2}}{1-x_{n \nu}^{2}} \frac{p_{n}^{2}(x)}{p_{n}^{\prime 2}\left(x_{n \nu}\right)\left(x-x_{n \nu}\right)^{2}} .
$$

Now setting

$$
\sum_{\nu=1}^{n}\left|\rho_{n \nu}(x)\right|=\sum_{x_{n \nu \leq 0}}\left|\rho_{n \nu}(x)\right|+\sum_{x_{n \nu}>0}\left|\rho_{n \nu}(x)\right|
$$

and considering the interval $-1<x \leqq 0$, we have for $x_{n \nu}$ 's $>0$,

$$
\left|x-x_{n \nu}\right|>\left|x_{n \nu}\right| \text {. }
$$

Thus from (9.2) and (8.10)

$$
\sum_{x_{n \nu}>0}\left|\rho_{n \nu}(x)\right| \leqq\left\{\begin{array}{c}
\frac{1}{16} \sum_{x_{n \nu}>0} \frac{1}{\left|x_{n \nu}\right|^{3}} \frac{\left(1-x^{2}\right)}{\left(1-x_{n \nu}^{2}\right)^{3 / 2}} \frac{\left[P_{m}^{(p,(q-1 / 2))}\left(2 x^{2}-1\right)\right]^{2}}{\left[\frac{d}{d t} P_{m}^{(p,(q-1 / 2))}(t)\right]_{t=2 x_{n \nu}^{2}-1}^{2}} \\
\text { for } n=2 m \\
\frac{1}{16} \sum_{x_{n \nu}>0} \frac{1}{x_{n \nu}{ }^{4}} \frac{\left(1-x^{2}\right)}{\left(1-x_{n \nu}^{2}\right)^{2}} \frac{\left[P_{m}^{(p,(q+1 / 2))}\left(2 x^{2}-1\right)\right]^{2}}{\left[\frac{d}{d t} P_{m}^{(p,(q+1 / 2))}(t)\right]_{t=2 x_{n \nu}^{2}-1}^{2}} \\
\text { for } n=2 m+1
\end{array}\right.
$$

which on account of (5.8) and the Lemmas 6.1 and 6.2, gives,

$$
\sum_{x_{n} \nu>0}\left|\rho_{n \nu}(x)\right| \leqq\left\{\begin{array}{r}
\sum_{\nu=1}^{m} O\left(n^{-1}\right) \frac{n^{3}}{\nu^{3}} \cdot \frac{\nu^{2 p+3}}{n^{2 p+4}}+\sum_{\nu=1}^{m} O\left(n^{-1}\right) \frac{n^{3}}{\nu^{3}} \cdot \frac{\nu^{q+2}}{n^{q+3}} \\
\text { for } n=2 m \\
\sum_{\nu=1}^{m} O\left(n^{-1}\right) \frac{n^{4}}{\nu^{4}} \cdot \frac{\nu^{2 p+3}}{n^{2 p+4}}+\sum_{\nu=1}^{m} O\left(n^{-1}\right) \frac{n^{4}}{\nu^{4}} \cdot \frac{\nu^{q+4}}{n^{q+5}} \\
\text { for } n=2 m+1
\end{array}\right.
$$

Since $0<p \leqq \frac{1}{2}$ and $0<q<1$, it follows from (9.4) that

$$
\sum_{x_{n \nu>0}}\left|\rho_{n \nu}(x)\right| \leqq O\left(n^{-\delta}\right) \quad-1<x \leqq 0
$$

where $\delta=\min (2 p, q)>0$.

Again let $x_{n \nu} \leqq 0,-1<x \leqq 0$ and

$$
\sum_{x_{n \nu} \leqq 0}\left|\rho_{n \nu}(x)\right|=\sum_{\substack{x, y \leq 0 \\\left|x-x_{n \nu}\right| \leqq n-\delta / 2}}\left|\rho_{n \nu}(x)\right|+\sum_{\substack{x_{n \nu} \leq 0 \\\left|x-x_{n \nu}\right|>n-\delta / 2}}\left|\rho_{n \nu}(x)\right|=\Sigma^{\prime}+\Sigma^{\prime \prime} .
$$


On account of (9.2) the following holds in the interval $-1<x \leqq 0$.

$$
\begin{aligned}
\Sigma^{\prime}\left|\rho_{n \nu}(x)\right| & \leqq n^{-\delta / 2} \Sigma^{\prime} \frac{\left(1-x^{2}\right) p_{n}^{2}(x)}{\left(1-x_{n \nu}^{2}\right) p_{n}^{\prime 2}\left(x_{n \nu}\right)\left(x-x_{n \nu}\right)^{2}} \\
& \leqq \frac{n^{-\delta / 2}}{|1-q|} \Sigma^{\prime} \frac{\left(1-x^{2}\right)}{\left(1-x_{n \nu}^{2}\right)} v_{n \nu}(x) \frac{p_{n}^{2}(x)}{p_{n}^{\prime 2}\left(x_{n \nu}\right)\left(x-x_{n \nu}\right)^{2}} \\
& \leqq \frac{n^{-\delta / 2}}{|1-q|} \Sigma^{\prime} r_{n \nu}(x) \leqq \frac{n^{-\delta / 2}}{|1-q|} .
\end{aligned}
$$

From (9.6) we have

$$
\Sigma^{\prime \prime}\left|\rho_{n \nu}(x)\right| \leqq n^{\delta / 2} \Sigma^{\prime \prime} \frac{\left(1-x^{2}\right)}{\left(1-x_{n_{\nu}}^{2}\right)} \frac{p_{n}^{2}(x)}{p_{n}^{\prime 2}\left(x_{n \nu}\right)} .
$$

But owing to (8.10), we have

$$
\Sigma^{\prime \prime}\left|\rho_{n \nu}(x)\right| \leqq\left\{\begin{array}{l}
\frac{n^{\delta / 2}}{16} \Sigma^{\prime \prime} \frac{1}{x_{n \nu}^{2}} \frac{\left(1-x^{2}\right)}{\left(1-x_{n \nu}^{2}\right)} \frac{p_{n}^{2}(x)}{\left[\frac{d}{d t} P_{m(t)}^{(p,(q-1 / 2))}\right]_{t=2 x_{n \nu}^{2}-1}} \text { for } n=2 m \\
\frac{n^{\delta / 2}}{16} \Sigma^{\prime \prime} \frac{\left(1-x^{2}\right)}{x_{n \nu}^{4}\left(1-x_{n \nu}^{2}\right)^{2}} \frac{p_{n}^{2}(x)}{\left[\frac{d}{d t} P_{m}^{(p,(q+1 / 2))}(t)\right]_{t=2 x_{n \nu}^{2}-1}^{2}} \text { for } n=2 m+1
\end{array}\right.
$$

which by (5.8), and Lemmas 6.1 and 6.2 gives

(9.8) $\quad \Sigma^{\prime \prime}\left|\rho_{n \nu}(x)\right|$

$$
\leqq \begin{cases}n^{\delta / 2}\left[\sum_{\nu=1}^{m} O\left(n^{-1}\right) \frac{n^{2}}{\nu^{2}} \frac{\nu^{2 p+3}}{n^{2 p+4}}+\sum_{\nu=1}^{m} O\left(n^{-1}\right) \frac{n^{2}}{\nu^{2}} \frac{\nu^{q+2}}{n^{q+3}}\right] & \text { for } n=2 m \\ n^{\delta / 2}\left[\frac{\left(1-x^{2}\right) p_{n}^{2}(x)}{p_{n}^{\prime 2}(0)}+\sum_{\nu=1}^{m} O\left(n^{-1}\right) \frac{n^{4}}{\nu^{4}} \frac{\nu^{2 p+3}}{n^{2 p+4}}+\sum_{\nu=1}^{m} O\left(n^{-1}\right) \frac{n^{4}}{\nu^{4}} \frac{\nu^{q+4}}{n^{q+5}}\right] & \text { for } n=2 m+1 .\end{cases}
$$

For $n=2 m+1$ we obtain by using $(6.2)$

$$
\frac{\left(1-x^{2}\right) p_{n}^{2}(x)}{p_{n}^{\prime 2}(0)}=\frac{\left(1-x^{2}\right) x^{2} P_{m\left(2 x^{2}-1\right)}^{2(p+1 / 2))}}{\left[P_{m(-1)}^{(p,(q+1 / 2)}\right]^{2}}=\frac{O\left(n^{-1}\right)}{\left(\begin{array}{c}
m+\frac{q+1}{2} \\
m
\end{array}\right)^{2}} .
$$

From this as well as from (9.8) we see that in the interval $-1<x \leqq 0$

$$
\sum_{\nu=1}^{n}\left|\rho_{n \nu}(x)\right| \leqq O\left(n^{-\delta / 2}\right) .
$$


Similarly it follows in the interval $0 \leqq x<1$ that

$$
\sum_{\nu=1}^{n}\left|\rho_{n \nu}(x)\right| \leqq O\left(n^{-\delta / 2}\right) .
$$

At $x= \pm 1$, the lemma obviously holds.

10. The proof of the Theorem 1. We now apply the usual argument. We have $S_{n}(x, f)$ our interpolating polynomial and $\Pi(x)$ an arbitrary polynomial of degree $2 n$ at most. Then there holds

$$
S_{n}(x, f)-f(x)=S_{n}(x, f-\Pi)+(\Pi(x)-f(x)) .
$$

From (2.1) and (1.11) we get

$$
S_{n}(x, f)-f(x)=\sum_{\nu=0}^{n+1}\left\{f\left(x_{n \nu}\right)-\Pi\left(x_{n \nu}\right)\right\} r_{n \nu}(x)+\sum_{\nu=0}^{n}\left(y_{n \nu}^{*}-\Pi^{\prime}\left(x_{n \nu}\right) \rho_{n_{\nu}}(x) .\right.
$$

Now by Weistrass approximation theorem for $-1 \leqq x \leqq 1$

$$
\Pi(x)-f(x)=o(1) .
$$

Now

$$
\begin{aligned}
& \left|\sum_{\nu=0}^{n+1}\left\{f\left(x_{n \nu}\right)-\Pi\left(x_{n \nu}\right)\right\} r_{n \nu}(x)\right| \\
\leqq & \max _{-1 \leqq x \leqq 1}|f(x)-\Pi(x)| \sum_{\nu=0}^{n+1}\left|r_{n \nu}(x)\right|=o(1)
\end{aligned}
$$

owing to (10.3) and Lemma 8.1

If $M=\max . \Pi^{\prime}(x)$ then in the interval $-1 \leqq x \leqq 1$

$$
\left|\sum_{\nu=1}^{n}\left(y_{n \nu}^{*}-\pi^{\prime}\left(x_{n \nu}\right)\right) \rho_{n \nu}(x)\right| \leqq\left(c n^{\eta}+M\right) \sum_{\nu=1}^{n}\left|\rho_{n \nu}(x)\right|=o(1)
$$

in consequence of Lemma 9.1 and $\left|\beta_{n \nu}\right| \leqq c n^{\eta}$, where $0 \leqq \eta<\frac{\delta}{2}<1$ and $\delta=(2 p, q)>0$.

Thus (10.2), (10.3), (10.4) and (10.5) complete the proof of our Theorem 1.

11. Proof of Theorem 2. The conjugate points belonging to our point-system owing to (4.6), (1.8) and Lemma 7.1 (i) are given by

$$
\begin{aligned}
X_{n \nu} & =x_{n \nu}+\frac{x_{n \nu}}{2\left\{\frac{p x_{n \nu}^{2}}{1-x_{n \nu}^{2}}-\frac{q}{2}\right\}} \\
& =x_{n \nu}\left[\frac{2 p+(1-2 p-q)\left(1-x_{n \nu}^{2}\right)}{2 p-(2 p+q)\left(1-x_{n \nu}^{2}\right)}\right] \quad x_{n \nu} \neq 0 .
\end{aligned}
$$


If however $x_{n \nu}=0$ i.e., in the case when $n=2 m+1$ and $\nu=$ $m+1$, then it follows from (4.6), (1.8) and Lemma 7.1(ii) that

$$
X_{2 m+1, m+1}=\infty \text {. }
$$

Now we shall make use of the following statements in the proof of Theorem 2.

Let $(\alpha, \beta)$ be a fixed part of the interval $[-1,1]$ but as small as we please. Consider the fundamental point system (4.3) or (4.4). We prove that for any value of $n$ sufficiently large at least one member of the series of triangular matrix of the fundamental point-system lies within the interval $(\alpha, \beta)$. Let

$$
f(x)=\left\{\begin{array}{l}
0 \text { for }-1 \leqq x<\alpha \\
(x-\alpha)(\beta-x) \text { for } \alpha \leqq x \leqq \beta \\
0 \text { for } \beta<x \leqq 1
\end{array}\right.
$$

Then $f(x)$ is apparently continuous in the interval $-1 \leqq x \leqq 1$. Let us assume that it is not so then there is a series $n_{1}<n_{2}<n_{3} \cdots<n_{i} \cdots$ such that no member of the point group belonging to these indices $x_{n i, 1}, x_{n i, 2}, \cdots, x_{n i n i}(i=1,2, \cdots)$ lie in the interval $(\alpha, \beta)$. Therefore in the interval $-1 \leqq x \leqq 1 \lim _{i \rightarrow \infty} S_{n i}(f, x)=0$ holds. On the otherhand according to Theorem 1 in place of $x=\alpha+\beta / 2$

$$
\lim _{i \rightarrow \infty} S_{n i}(f, x)=f\left(\frac{\alpha+\beta}{2}\right)=\left(\frac{\alpha-\beta}{2}\right)^{2} \neq 0
$$

contradicts the foregoing inference, i.e., point-system (4.3) or (4.4) lie thickly in the interval $-1 \leqq x \leqq 1$. It can also be proved that the conjugate point-system belonging to (4.3) or (4.4) thickly cover the interval $-1 \leqq x \leqq 1$.

The conjugate points belonging to points $x_{n \nu} \neq 0$ can according to (11.1) be obtained from the function

$$
g(x)=x\left[\frac{1-q-(1-2 p-q) x^{2}}{(2 p+q) x^{2}-q}\right]
$$

in the places $x_{n \nu}$. In the interval $-1 \leqq x \leqq 1, g^{\prime}(x)<0$. Therefore the function $g(x)$ in the interval $(-\sqrt{q / 2 p+q}, \sqrt{q / 2 p+q})$ which on account of $0<p \leqq \frac{1}{2}$ and $0<q<1$ forms a part interval of $[-1,1]$ diminishes continuously, is continuous and its value includes all values from $+\infty$ to $-\infty$. There must also be two points $a_{1}$ and $b_{1}$ different from each other within the interval $[-\sqrt{q / 2 p+q}, \sqrt{q / 2 p+q}]$ so that $g\left(a_{1}\right)=-1$ and $g\left(b_{1}\right)=1$. Since $g^{\prime}(x)<0$ it follows that $-1 \leqq g(x) \leqq 1$ holds in the interval $b_{1} \leqq x \leqq a_{1}$. Let $a_{2}$ and $b_{2}$ be again two different real values for which $-1<a_{2}<b_{2}<1$ holds. 
Then there must obviously lie in the interval $\left(a_{1}, b_{1}\right)$ two different points $a_{3}$ and $b_{3}$ such that $g\left(a_{3}\right)=a_{2}$ and $g\left(b_{3}\right)=b_{2}$. Since we have already proved that at least one point of each series of the point-system (4.3) or (4.4) must belong to the index $n$ within the interval $\left(a_{3}, b_{3}\right)$. Therefore it follows that the conjugate points belonging to the fundamental points lying within the interval $(\alpha, \beta)$ must owing to monotony of $g(x)$ from this index onwards lie within the interval $\left(a_{2}, b_{2}\right), a_{2}$ and $b_{2}$ can lie as near to each other as we please. Thus Theorem 2 is proved.

\section{REFERENCES}

1. J. Balázs, On the convergence of Hermite-Fejér interpolation process, Acta. Math. Acad. Sci. Hungar, 9 (1958), 363-377.

2. E. Egerváry, and P., Turan, Notes on interpolation $V$, Acta Math. Acad. Sci. Hunger. 9 (1958), 259-267.

3. L. Fejér, Lagrangesche interpolation und die zugehoriegen konjugiertch Punkte, Math. Annalen 106 (1932), 1-55.

, On the characterization of some remarkable systems of points of interpolation by means of conjugate points, Amer. Math. Monthly 41 (1934), 1-14.

5. K. V. Laščenov, On a class of orthogonal polynomial, Leningrad Gos. Ped. Inst. Zap. 89 (1953), 167-187.

6. P. Szász, On Quasi-Hermite-Fejer interpolation., Acta Math. Acad. Sci. Hungar., 10 (1959), 413-439.

7. G. Szegö, Orthogonal polynomials, Amer. Math. Soc., Colloquium Publications. Vol. 23.

Received September 12, 1962, and in revised form May 12, 1964.

Department of Mathematics and Astronomy

THE UNIVERSITY, LUCKNOW, INDIA 



\section{PACIFIC JOURNAL OF MATHEMATICS}

\section{EDITORS}

\author{
H. SAMELSON \\ Stanford University \\ Stanford, California \\ J. P. JANS \\ University of Washington \\ Seattle, Washington 98105
}

\section{J. DugundJI}

University of Southern California Los Angeles, California 90007

RICHARD ARENS

University of California

Los Angeles, California 90024

\section{ASSOCIATE EDITORS}
E. F. BECKENBACH
B. H. NeumanN
F. WOLF
K. YOSIDA

\section{SUPPORTING INSTITUTIONS}

\author{
UNIVERSITY OF BRITISH COLUMBIA \\ CALIFORNIA INSTITUTE OF TECHNOLOGY \\ UNIVERSITY OF CALIFORNIA \\ MONTANA STATE UNIVERSITY \\ UNIVERSITY OF NEVADA \\ NEW MEXICO STATE UNIVERSITY \\ OREGON STATE UNIVERSITY \\ UNIVERSITY OF OREGON \\ OSAKA UNIVERSITY \\ UNIVERSITY OF SOUTHERN CALIFORNIA
}

\author{
STANFORD UNIVERSITY \\ UNIVERSITY OF TOKYO \\ UNIVERSITY OF UTAH \\ WASHINGTON STATE UNIVERSITY \\ UNIVERSITY OF WASHINGTON \\ AMERICAN MATHEMATICAL SOCIETY \\ CHEVRON RESEARCH CORPORATION \\ TRW SYSTEMS \\ NAVAL ORDNANCE TEST STATION
}

Mathematical papers intended for publication in the Pacific Journal of Mathematics should be typewritten (double spaced). The first paragraph or two must be capable of being used separately as a synopsis of the entire paper. It should not contain references to the bibliography. Manuscripts may be sent to any one of the four editors. All other communications to the editors should be addressed to the managing editor, Richard Arens at the University of California, Los Angeles, California 90024 .

50 reprints per author of each article are furnished free of charge; additional copies may be obtained at cost in multiples of 50 .

The Pacific Journal of Mathematics is published monthly. Effective with Volume 16 the price per volume (3 numbers) is $\$ 8.00$; single issues, $\$ 3.00$. Special price for current issues to individual faculty members of supporting institutions and to individual members of the American Mathematical Society: $\$ 4.00$ per volume; single issues $\$ 1.50$. Back numbers are available.

Subscriptions, orders for back numbers, and changes of address should be sent to Pacific Journal of Mathematics, 103 Highland Boulevard, Berkeley 8, California.

Printed at Kokusai Bunken Insatsusha (International Academic Printing Co., Ltd.), No. 6, 2-chome, Fujimi-cho, Chiyoda-ku, Tokyo, Japan.

PUBLISHED BY PACIFIC JOURNAL OF MATHEMATICS, A NON-PROFIT CORPORATION

The Supporting Institutions listed above contribute to the cost of publication of this Journal, but they are not owners or publishers and have no responsibility for its content or policies. 


\section{Pacific Journal of Mathematics \\ Vol. 20, No. $2 \quad$ October, 1967}

Edward Dewey Davis, Ideals of the principal class, $R$-sequences and a certain monoidal transformation ............................. 197

Richard Mansfield Dudley, Sub-stationary processes ................ 207

Newton Seymour Hawley and M. Schiffer, Riemann surfaces which are doubles of plane domains ......................... 217

Barry E. Johnson, Continuity of transformations which leave invariant certain translation invariant subspaces ................... 223

John Eldon Mack and Donald Glen Johnson, The Dedekind completion of

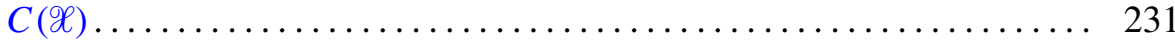

K. K. Mathur and R. B. Saxena, On the convergence of quasi-Hermite-Fejér interpolation................................... 245

H. D. Miller, Generalization of a theorem of Marcinkiewicz............ 261

Joseph Baruch Muskat, Reciprocity and Jacobi sums ............... 275

Stelios A. Negrepontis, On a theorem by Hoffman and Ramsay .......... 281

Paul Adrian Nickel, A note on principal functions and multiply-valent canonical mappings .............................. 283

Robert Charles Thompson, On a class of matrix equations ............. 289

David Morris Topping, Asymptoticity and semimodularity in projection lattices ........................................ 317

James Ramsey Webb, A Hellinger integral representation for bounded linear functionals

Joel John Westman, Locally trivial $C^{r}$ groupoids and their representations...

Hung-Hsi Wu, Holonomy groups of indefinite metrics 